Check for updates

Cite this: RSC Adv., 2017, 7, 45145

Received 30th July 2017

Accepted 15th September 2017

DOI: $10.1039 / c 7 r a 08391 b$

rsc.li/rsc-advances

\section{Physicochemical characterization of high-quality bacterial cellulose produced by Komagataeibacter sp. strain W1 and identification of the associated genes in bacterial cellulose production $\dagger$}

\author{
Shan-Shan Wang, $t^{\mathrm{ab}}$ Yong-He Han, (D) $t^{\mathrm{b}}$ Yu-Xuan Ye, ${ }^{\mathrm{c}}$ Xiao-Xia Shi, ${ }^{\mathrm{c}}$ Ping Xiang, (D) \\ Deng-Long Chen ${ }^{\star b d}$ and Min Li ${ }^{\star a}$
}

\begin{abstract}
In the last few decades, bacteria capable of bacterial cellulose (BC) synthesis and the characterization of BC have been well-documented. In this study, a new BC-producing bacterial strain was isolated from fermented vinegar. The BC morphology, composition and diameter distribution, and the genes associated with $\mathrm{BC}$ production were analyzed. The results showed that one out of five isolates belonging to Komagataeibacter was a BC-producer, which mostly produced the typical cellulose I consisting of nanofibrils and had several functional groups similar to typing paper (i.e., plant cellulose). Several known genes such as glk, pgm and UPG2 in glucose metabolisms, bcsA, bcsB, bcsC and bcsD in BC synthesis and cmcax, ccpAx, bglxA and other genes in BC synthesis regulation or c-di-GMP metabolisms have also been found in the strain W1 based on genome sequencing and gene annotations. The functions of BcsX and BcxY might also be important for BC synthesis in Komagataeibacter sp. W1. Our study provided a new $B C$-producing bacterial strain that could be used to prepare high-quality $B C$ and to study $B C$ synthesis mechanisms.
\end{abstract}

\section{Introduction}

Cellulose is an organic compound that acts as a basic structural material of most plants and often combines with hemicellulose, lignin and pectin. ${ }^{1}$ Although plant-derived cellulose has been used in several areas such as paper-making, all-cellulose composite preparation and dietary fiber intake, ${ }^{2}$ it needs to be purified by enzymatic and/or mechanical treatments before further use. ${ }^{1,3}$ In addition to the high cost, these processes may also change the cellulose performance and limit its applications in several areas. ${ }^{1}$ Microorganisms including algae (e.g., Cladophora and Vallonia), fungi (e.g., Dictyostelium and Saprolegnia) and bacteria (e.g., Acetobacter, Achromobacter, Acidomonas, Aerobacter, Agrobacterium, Alcaligenes, Ameyamaea, Asaia,

${ }^{a}$ College of Life Science, Fujian Normal University, Fuzhou, 350108, China. E-mail: mli@fjnu.edu.cn

${ }^{b}$ Quangang Petrochemical Research Institute, Fujian Normal University, Quanzhou, 362801, China.E-mail: dlchen@fjnu.edu.cn; chendenglong@163.com

'State Key Laboratory of Pollution Control and Resource Reuse, School of the Environment, Nanjing University, Jiangsu, 210023, China

${ }^{a}$ College of Environmental Science and Engineering, Fujian Normal University, Fuzhou, 350108, China

$\dagger$ Electronic supplementary information (ESI) available. See DOI: $10.1039 / \mathrm{c} 7 \mathrm{ra} 08391 \mathrm{~b}$

\$ These two authors contributed equally to this work and should be considered co-first authors.
Gluconobacter, Gluconacetobacter, Granulibacter, Komagataeibacter, Kozakia, Neoasaia, Neokomagataea, Pseudomonas, Rhizobium, Saccharibacter, Sarcina, Swaminathania, Tanticharoenia and Zoogloea $)^{4-6}$ also produce cellulose and have attracted people's attention due to its higher purity, crystallinity and water-holding capacity, lower production cost, thinner fiber diameter (i.e., $\sim 30-50 \mathrm{~nm}$ ) and better biocompatibility as compared to plant cellulose. ${ }^{7,8}$ Now bacterial cellulose (BC) has been widely used as food material, paper-making material, acoustic or filter membrane, cosmetic and clothing material, medical material and electronic paper substrate.

It is hypothesized that cellulose production in bacteria helps them to move to the oxygen-rich medium surface, protects them from ultraviolet light and retains moisture, and establishes close contact with a preferred host to facilitate efficient hostbacteria interactions. ${ }^{9,10}$ Based on molecular evidence, the synthesis of BC is a multistep process including two main mechanisms: the synthesis of uridine diphosphoglucose (UDPGlc), followed by the polymerization of glucose into long and unbranched chains, i.e., $\beta-1,4-\mathrm{D}-\mathrm{glucan}$, associated with hydrogen bonding. ${ }^{1,7}$ While the former mechanism is well known, the latter still needs exploring.

As the most two important members of BC producers, both Gluconacetobacter and Komagataeibacter are formerly grouped into Acetobacter and now regrouped into independent genera based on 16S rRNA gene sequences and phenotypic, ecologic 
and chemotaxonomic characteristics. ${ }^{11}$ Studies have shown that Gluconacetobacter is the most-well known genus due to its highest BC production ability among the reported BCproducers, ${ }^{1,7}$ while Komagataeibacter contributes mostly to the rapid $\mathrm{pH}$ decrease and $\mathrm{BC}$ production during vinegar fermentation. ${ }^{12,13}$ Although more than 14 species of Komagataeibacter have been regrouped from Gluconacetobacter, ${ }^{\mathbf{1 4 , 1 5}}$ their $\mathrm{BC}$ production characteristics warrant further investigations. For example, most studied BC-encoding genes are $b c s A, b c s B, b c s C$ and $b c s D$, but the functions of other genes such as $b c s X$ and $b c s Y$ are little known. ${ }^{16} \mathrm{~A}$ recent study showed that $g q q A$ encoded a novel protein involving in bacterial quorum quenching and cellulose formation, indicating more attention should be paid to $\mathrm{BC}$ synthesis mechanisms in bacteria. ${ }^{17}$

Since BC production is naturally occurring, most BCproducing bacteria are often isolated from the carbohydratecontaining substances such as rotten fruits, kombucha and vinegar. ${ }^{16,18,19}$ In the present study, a bacterium belonging to the genus Komagataeibater was firstly isolated from a famous vinegar factory located in Yongchun, Quanzhou, China. By subjecting the strain to Hestrin and Schramm (HS) medium, a typical $\mathrm{BC}$ membrane was found and characterized by scanning electron microscopy (SEM), X-ray diffraction (XRD) and Fourier transform infrared (FTIR) spectroscopy. Although the genomes of several species of Komagataeibacter have been sequenced, the gene information associated with BC synthesis and regulation is little known except for $K$. nataicola. ${ }^{6}$ To identify the genes involving in glucose utilization and BC synthesis, draft genome sequencing and associated annotations (e.g., COG functional prediction, NR annotation and Swissprot annotation) were also conducted in this study. The fully aim of this study was to give more precise work on understanding of BC synthesis mechanisms in bacteria and its importance in current and future applications.

\section{Materials and methods}

\subsection{Sampling and bacteria isolation}

To isolate BC-producing bacteria, both the vinegar and $\mathrm{BC}$ membranes were collected from Yongchun Laocu Industry Co., Ltd and stored in sterile plastic bottles (Corning, USA) in ice bath for taking back to lab. Before use, the BC membranes were washed carefully by sterile Milli-Q water to remove the surface bacteria, followed by soaking in $20 \mathrm{~mL}$ PBS containing $0.85 \%$ $\mathrm{NaCl}^{20}$ and shaking at $200 \mathrm{rpm}$ for $30 \mathrm{~min}$. After that, aliquots of vinegar and washing buffer were streaked onto HS agar medium consisting of $2 \%$ glucose, $0.5 \%$ peptone, $0.5 \%$ yeast extract, $0.68 \% \quad \mathrm{Na}_{2} \mathrm{HPO}_{4} \cdot 12 \mathrm{H}_{2} \mathrm{O}, \quad 0.051 \% \quad \mathrm{MgSO}_{4} \cdot 7 \mathrm{H}_{2} \mathrm{O}, \quad 0.115 \%$ $\mathrm{C}_{6} \mathrm{H}_{8} \mathrm{O}_{7} \cdot \mathrm{H}_{2} \mathrm{O}$ and $1.8 \%$ agar $(\mathrm{pH}$ was adjusted to 6.0 by $1 \mathrm{M}$ $\mathrm{NaOH}$ and $\mathrm{HCl}$ ). ${ }^{21}$ All isolates were purified by re-streaking onto HS agar medium three times and subjected to HS liquid medium following $7 \mathrm{~d}$ incubation to pick out the bacteria capable of BC production. The obtained BC was pretreated as previously described and the BC-producing bacteria in the supernatant were mixed with $30 \%$ glycerol by $1: 1(\mathrm{v} / \mathrm{v})$ and stored at $-80{ }^{\circ} \mathrm{C}$ or re-streaked onto HS agar medium following $7 \mathrm{~d}$ incubation and stored at $4{ }^{\circ} \mathrm{C}$ for further use.

\subsection{Bacterial identification}

All isolates that could grew on HS agar medium were collected and re-cultivated in HS liquid medium for $7 \mathrm{~d}$, followed by centrifugation at $8000 \times g$ for $5 \mathrm{~min}$. The collected bacterial biomass was used to extract total DNA by using a FastDNA ${ }^{\circledR}$ SPIN Kit for Soil (MP Biomedicals, USA) according to the manufacturer's instructions. The 16S rRNA gene was amplified by the universal primers 27F (AGAGTTTGATCCTGGCTCAG) and 1492R (ACGGCTACCTTGTTACGACTT) on a T100 Thermocycler (BioRad, USA). ${ }^{20}$ The polymerase chain reaction (PCR) mixtures consisted of $12.5 \mu \mathrm{L}$ of $2 \times$ Mix (Yifeixue Biotechnology, Nanjing, China), $1.5 \mu \mathrm{L}$ of primer pair $(10 \mu \mathrm{M}), 10 \mu \mathrm{L}$ of PCR degrade water and $1 \mu \mathrm{L}$ of DNA template. The PCR programs for $16 \mathrm{~S}$ rRNA amplification were: $5 \mathrm{~min}$ at $94{ }^{\circ} \mathrm{C}$ for predenaturation, $35 \mathrm{~s}$ at $94{ }^{\circ} \mathrm{C}$ for denaturation, $30 \mathrm{~s}$ at $55{ }^{\circ} \mathrm{C}$ for annealing and $1.5 \mathrm{~min}$ at $72{ }^{\circ} \mathrm{C}$ for extension (35 cycles), and $10 \mathrm{~min}$ at $72{ }^{\circ} \mathrm{C}$ for a final extension. ${ }^{20}$ The PCR products were purified and sequenced by GenScript Co., Ltd. (Nanjing, China). Based on the sequence analysis by BLAST similarity search in the NCBI database, the phylogenetic tree was constructed by using the neighbor-joining algorithm in the MEGA 5.0 program.

To have an observation of bacterial morphology, the biomass was pretreated by a sequential dehydration procedure and freeze-dried for $24 \mathrm{~h}$ (FreeZone 6 plus, Labconco, USA) according to Wang et al. ${ }^{20}$ After a spray-gold treatment, the morphology was observed by SEM (Quanta ${ }^{\mathrm{TM}} 250$ FEG, FEI, USA).

\subsection{Bacterial cellulose characterization by SEM, XRD and FTIR}

To observe the porous structure and fibril distribution on BC, one colony of the strain W1 was inoculated in HS liquid medium for $7 \mathrm{~d}$, followed by washing and shaking treatments as shown above. After centrifugation at $8000 \times g$ for $5 \mathrm{~min}$, the bacterial biomass was re-suspended in $10 \mathrm{~mL}$ sterile Milli-Q water, aliquots of which were transferred to a new medium to a final $\mathrm{OD}_{600}$ at 0.01 . After $7 \mathrm{~d}$ of incubation, the $\mathrm{BC}$ membranes were collected and washed by Milli-Q water, followed by boiling at $100{ }^{\circ} \mathrm{C}$ for $2 \mathrm{~h}$ by $0.1 \mathrm{M} \mathrm{NaOH}$ and another $2 \mathrm{~h}$ by Milli-Q water. The pre-treated BC was soaked in sterile Milli-Q water overnight at room temperature and then ovendried at $50{ }^{\circ} \mathrm{C}$. The dried $\mathrm{BC}$ was treated by spray-gold and observed by SEM. To evaluate the diameter distribution of BC fibrils, the SEM image of BC was analyzed on a NanoMeasurer 1.2 by calculating 100 nanofibrils randomly. In this study, the difference of each group of fibrils was $10 \mathrm{~nm}$, and the results were presented as \% of the total fibril numbers.

Similar to SEM, all samples used for further characterization by XRD (Bruker D8 ADVANCE, Germany) and FTIR (Thermo Scientific Nicolet iS5, USA) were pre-treated as described. Of which, XRD pattern was obtained using nickel filtered copper $K_{\alpha}$ radiation, with $0.1^{\circ}$ step, from $4^{\circ}$ to $70^{\circ}$ ( $2 \theta$, angle), while FTIR analysis was conducted in the attenuated total reflection (ATR) mode with 32 scans per measurement between 400 and $4000 \mathrm{~cm}^{-1}$. 


\subsection{Draft genome sequencing and key functional genes annotation}

To have an overview of BC synthesis mechanisms in the strain W1, the draft genome sequence and key functional genes were analyzed by Majorbio, Shanghai, China. Before sequencing, the total DNA was extracted as previously described and the DNA quality was evaluated by Nanodrop ${ }^{\circledR}$ ND-1000 (NanoDrop Technologies, Wilmington, DE, USA) based on DNA concentration and $\mathrm{OD}_{260} / \mathrm{OD}_{280}$. The established sample was fragmented to $400-500$ bp by using M220 Focused-ultrasonicator $^{\mathrm{TM}}$ (Covaris, Woburn, MA, USA), followed by library construction by using TruSeq ${ }^{\mathrm{TM}}$ DNA Sample Prep Kit (Illumina, Inc., USA) and sequencing on an Illumina Miseq (PE) platform by MiSeq Reagent KiT v3 (Illumina, Inc., USA). After data transformation by Base Calling, the raw sequences were saved as FASTQ and deposited in the NCBI-Sequence Read Archive (SRA) database (http://www.ncbi.nlm.nih.gov/Traces/ sra) with the accession numbers PRJNA388252 (BioProject number), SAMN07173612 (BioSample number) and SRP108180 (SRA Study number).

Before genome assembly, the low-quality data in the raw sequences were removed based on the following criteria: (1) the adapter sequences, (2) the sequences include A, G, C or T base in the $5^{\prime}$ terminal, (3) the terminal sequences with low quality values $(<20)$, (4) the reads include $\geq 10 \% \mathrm{~N}$ and (5) the small sequences $(<25 \mathrm{bp}$ ) after removing the adapter and lowquality data. The genome assembly was performed on a SOAP denovo platform (v2.04, http://soap.genomics.org.cn/) by multi-optimization of K-mer parameters, followed by a further base-proofreading by GapCloser (v1.12). ${ }^{22}$ The genome prediction was conducted by Glimmer (v3.02, http:// www.cbcb.umd.edu/software/glimmer/). For functional gene annotation, all protein sequences in correspond to the predicted genes were analyzed by blastp research (BLAST 2.2.28+) against the known sequences in Nr, String, Genes and GO databases. Of which, the String annotation can obtain COG function analysis information, i.e., the protein function, classification and evolution status. Moreover, the blast results from Genes database can assign a certain gene to a KO number in KEEG pathway.

\section{Results and discussion}

\subsection{Taxonomic characterization of BC-producing bacterial isolate}

By using the typical HS agar medium for isolation of BCproducing bacteria, a total of five strains were obtained. However, only one (i.e., strain $\mathrm{W} 1$ ) produced BC in HS liquid medium. As shown in Fig. 1A, limited colonies of the strain W1 were found on HS agar medium, which might be due to the fact that BC-producing bacteria often use $\sim 50 \%$ energy to produce $\mathrm{BC}$ as compared to normal bacteria that use most energy to reproduce offspring. ${ }^{23}$ In addition, the strain W1 formed unregular colonies that might contain $\mathrm{BC}$ as the colonies were tough and smooth on agar medium (Fig. 1A). SEM observation found that the cells of strain W1 were short rod-shaped without flagellum (Fig. 1B). The size of each cell was $1.58 \pm 0.11 \mu \mathrm{m}$ long by $0.91 \pm 0.09 \mu \mathrm{m}$ wide (Fig. 1B).

To further identify the strain W1, its $16 \mathrm{~S}$ rRNA sequence (GenBank accession number MF187480) was aligned with the known sequences retrieved from the NCBI database. All three groups that were often confused with each other previously were listed. The results showed that although the 16S rRNA sequence of W1 shared high similarity with most reference sequences, it belonged to the Group I, i.e., the genus Komagataeibacter (Fig. 2). However, the strain hadn't been exactly classified at species level, thereby being named as Komagataeibacter sp. W1 (Fig. 2). Since Komagataeibacter is regrouped from the genus Gluconacetobacter, a typical and widely-studied BC-producing family, the strain isolated in this study could be a good candidate for BC preparation. ${ }^{\mathbf{1 4}, 15}$ Studies also showed that Komagataeibacter contributed mostly to $\mathrm{pH}$ decrease in vinegar production, ${ }^{\mathbf{1 2}, \mathbf{1 3}}$ indicating that the strain $\mathrm{W} 1$ was an important member in vinegar fermentation and BC production in the sampling site.

\subsection{Morphological characteristics of cellulose produced by Komagataeibacter sp. strain W1}

As shown above, the strain $\mathrm{W} 1$ produced $\mathrm{BC}$ membranes, which were pale yellow and transparent before and after treatment in $0.1 \mathrm{M} \mathrm{NaOH}$ bath, respectively (Fig. 3A-D). To have an in situ observation of the $\mathrm{BC}$ with or without $\mathrm{NaOH}$ treatment, all
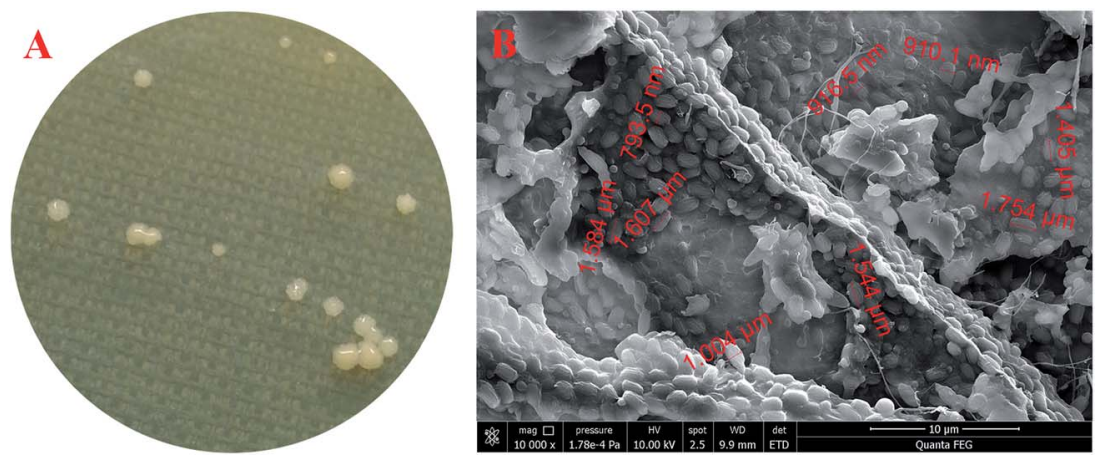

Fig. 1 Colony morphology (A) and SEM observation ((B), $10000 \times)$ of Komagataeibacter sp. W1 isolated from spiced vinegar fermentation tank in Yongchun, Quanzhou, Fujian, China. 


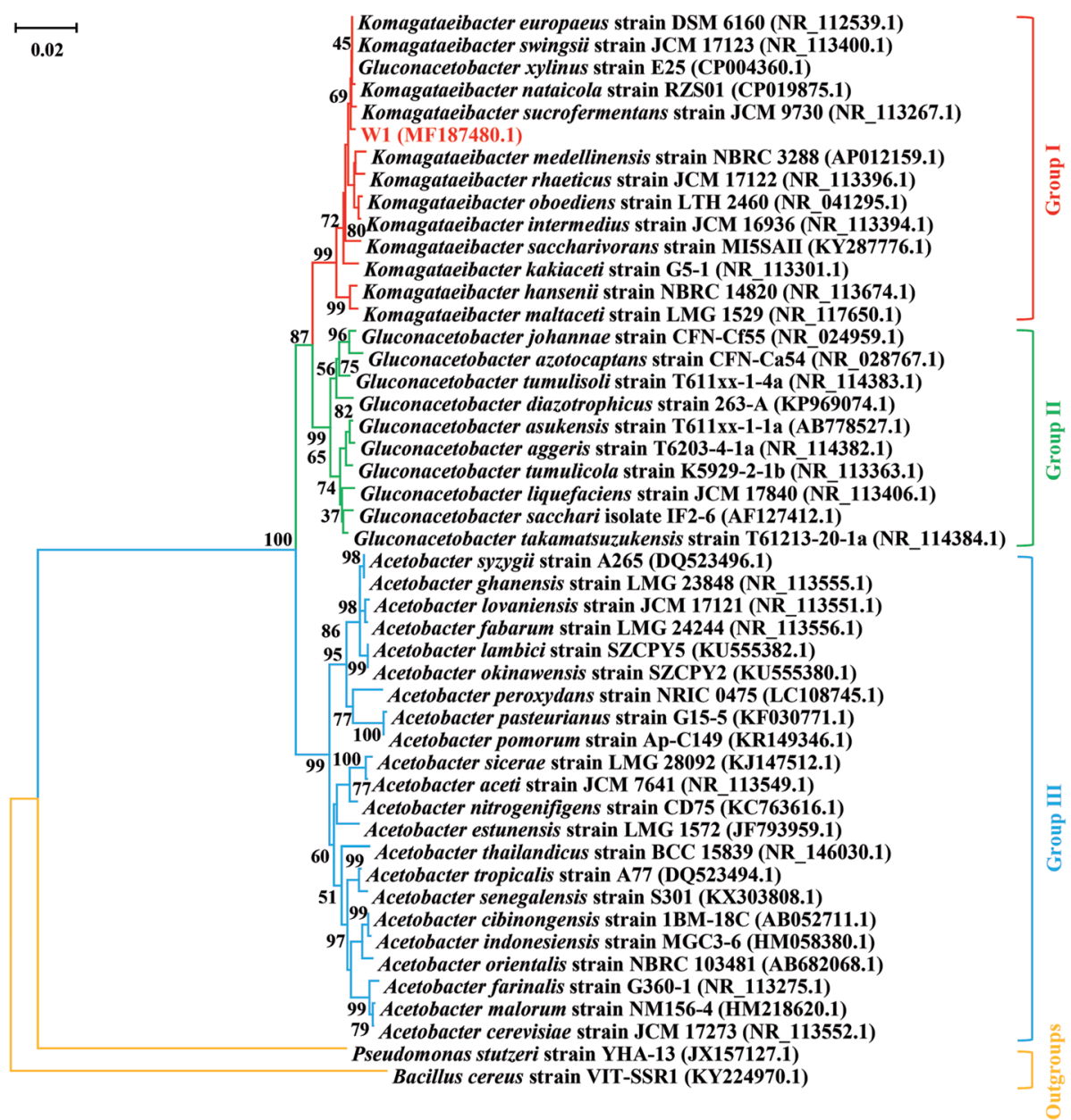

Fig. 2 Neighbor-joining phylogenetic trees of bacterial 16S rRNA sequence of Komagataeibacter sp. W1 retrieved from the fermentation tank of spiced vinegar in Yongchun, Quanzhou, Fujian, China. Reference sequences were retrieved from NCBI database. The tree root was constructed with bootstrap values calculated from 1000 resamplings. The numbers at each node indicate the percentage of bootstrap supporting. Scale bars indicate 20 mutations per 1000 bases. Group I, II and III were often confused with each other previously and have been classified into three independent groups recently.

samples were oven-dried and BC images were taken by SEM. Results showed that the $\mathrm{BC}$ membranes had a threedimensional structure and the bacteria distributed both on the surface of and inside BC membranes (Fig. 3E-L). It was also apparent that the bacteria produced fibrils on the cell surface (Fig. 3H), where several terminal complexes consist of BC synthase catalytic subunits, by a three-way branching pattern..$^{23,24}$ In this case, a single cell can produce a ribbon of $\mathrm{BC}$ with 10-100 microfibrils. ${ }^{23}$ As a result of cell mitosis, the fibril assembly occurs, forming the glucan chain sheets by van der Waals bonding followed by stacking of the sheets by H-bonding to form the crystalline structure, ${ }^{25,26}$ which were supported by our data as shown in Fig. 3L. We hypothesized that the strain W1 produced a typical BC with high purity (Fig. 3I-L).

To test the diameter distribution of the $\mathrm{BC}$ fibrils produced by Komagataeibacter sp. W1, 100 fibrils on SEM image were selected randomly and calculated by NanoMeasurer 1.2. As expected, $98 \%$ of the fibrils had the diameter $<100 \mathrm{~nm}, 77 \%$ of which were $<50 \mathrm{~nm}$ (Fig. 4), indicating that the BC obtained in this study consisted of nanofibrils. This was in line with previous studies that the visible BC fibrils consist of 40-60 nm cellulose ribbons, which are assembled by microfibrils consisting of 3-4 $\mathrm{nm}$ subelementary fibrils..$^{27,28}$

\subsection{XRD and FTIR analyses of cellulose produced by Komagataeibacter sp. strain W1}

Based on a SEM observation, the BC produced by Komagataeibacter sp. strain $\mathrm{W} 1$ had some typical characteristics of the known BC, e.g., including nanofibrils and pellicles (Fig. 3). To further verify the $\mathrm{BC}$ compositions and purity, XRD and FTIR analyses were also performed.

Fig. 5 shows XRD pattern of the BC produced by the strain W1. Similar to previous studies, three typical diffraction peaks were observed at $2 \theta 14.5^{\circ}, 16.6^{\circ}$ and $22.7^{\circ}$ (Fig. 5), corresponding to (110), (110), and (200) planes, respectively. ${ }^{29,30}$ Studies have shown that the broad peaks at $2 \theta \quad 14.5^{\circ}$ and $22.7^{\circ}$ are associated with the presence of cellulose $I_{\alpha}$ and cellulose $I_{\beta}$ phases, i.e., $100_{1 \alpha}, 110_{1 \beta}$ and $010_{1 \beta}$ planes at $14.5^{\circ}$ and $110_{1 \alpha}$ and $200_{1 \beta}$ at $22.7^{\circ} .31,32$ Thus, we concluded that W1-produced 

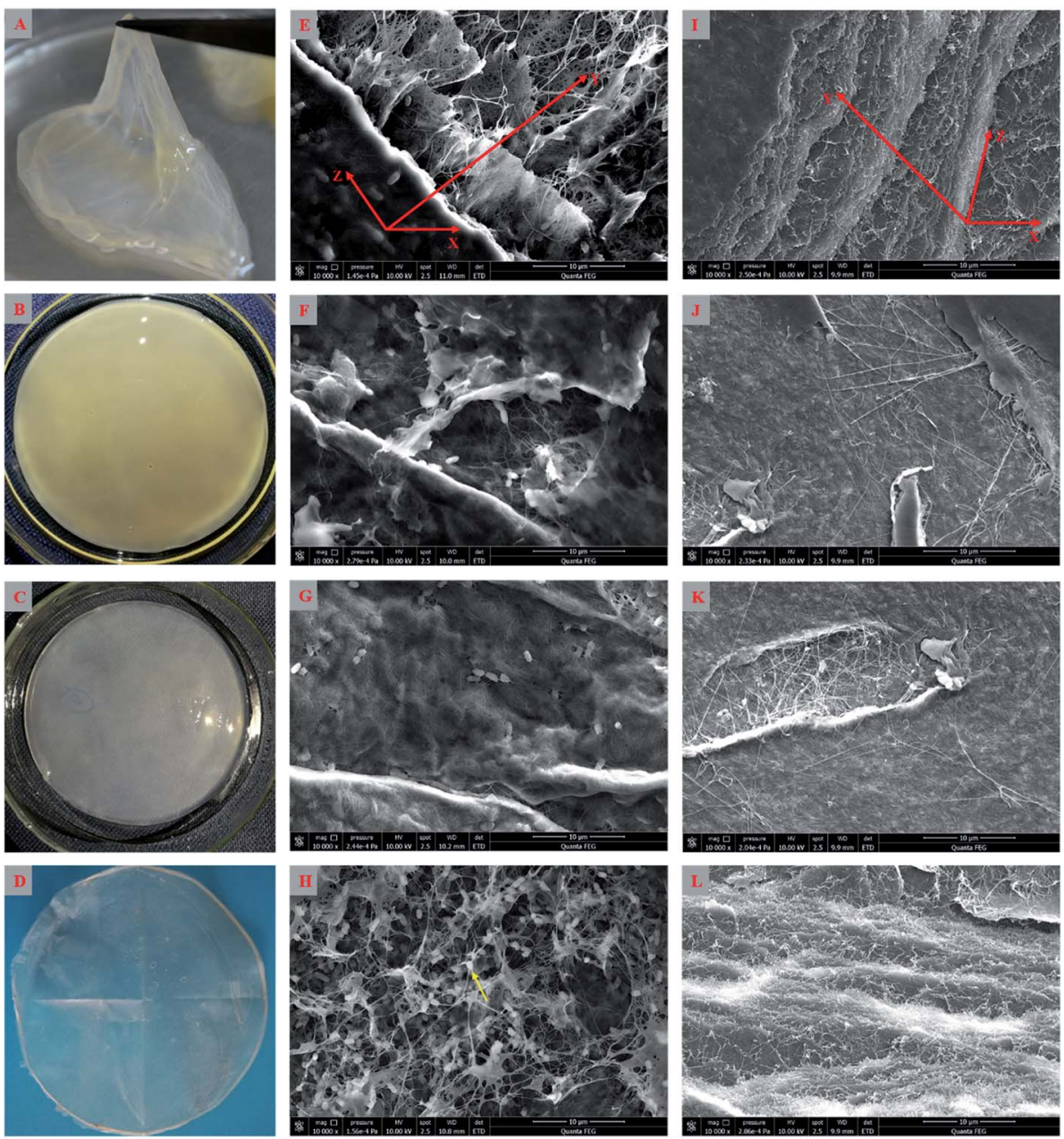

Fig. 3 The appearance (A-D) and SEM images (E-L) of the bacterial cellulose membranes produced by Komagataeibacter sp. W1. (A and B) indicate the samples without the treatment in $0.1 \mathrm{M} \mathrm{NaOH}$ bath for $2 \mathrm{~h}$; ( $\mathrm{C}$ and $\mathrm{D}$ ) are the samples with the treatment in $0.1 \mathrm{M} \mathrm{NaOH}$ bath for $2 \mathrm{~h}$ before and after oven-drying at $50{ }^{\circ} \mathrm{C}$, respectively; $(\mathrm{E}-\mathrm{H})$ indicate the bacterial distribution on the surface of and inside bacterial cellulose membranes, i.e., (E) full observation, (F) surface bacterial membranes, (G) bacterial distribution on the horizontal scale and (H) three-dimensional structure of the bacterial cellulose containing bacteria, of which the yellow arrow indicates the bacterial cellulose production and secretion site locating on the cell surface; $(\mathrm{I}-\mathrm{L})$ indicate the horizontal and cross-sectional observations of the bacterial cellulose with additional treatment in $0.1 \mathrm{M} \mathrm{NaOH}$ bath for $2 \mathrm{~h}$, i.e., (I) full observation, (J) horizontal surface textile structure of bacterial cellulose, (K) inter-horizontal textile structure of bacterial cellulose and $(\mathrm{L})$ three-dimensional-cross-sectional structure of the bacterial cellulose. Red arrows indicate three-dimension structure of the bacterial cellulose.

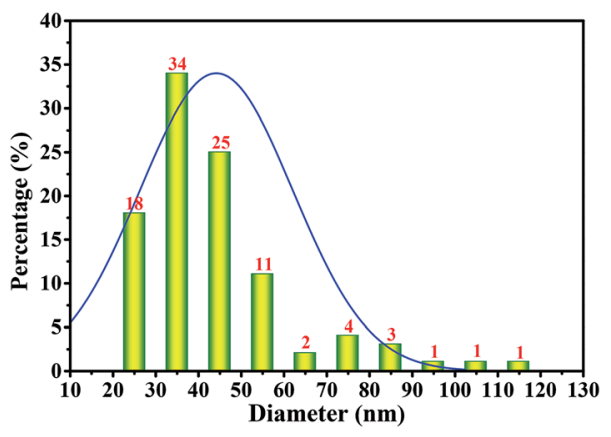

Fig. 4 Diameter distribution of the bacterial cellulose fibrils produced by Komagataeibacter sp. W1. The diameter calculation was performed on Nano Measurer 1.2 by calculating 100 nanofibrils randomly on SEM image of the bacterial cellulose membranes (50 000x).

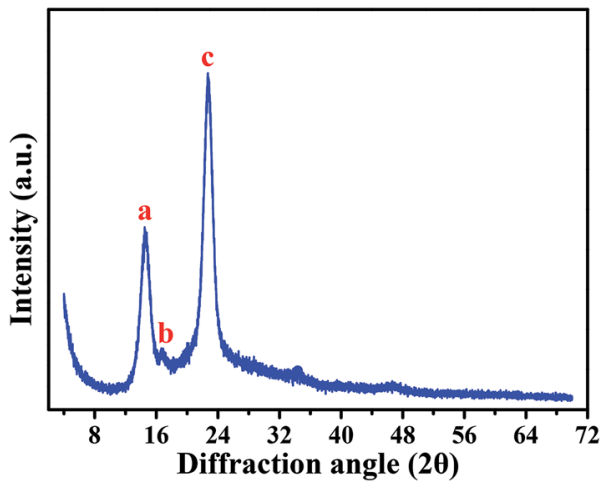

Fig. 5 XRD analysis of pure bacterial cellulose produced by Komagataeibacter sp. W1. 
BC was pure. However, similar to other studies, the broad diffraction peaks with sharpness observed in this study indicated that the products included semi-crystalline BC (Fig. 5). ${ }^{29,30}$

In addition to XRD characterization, FTIR analysis is also an important alternative to identify the types and purity of BC. ${ }^{24,33}$ To have an comparative view on functional groups in cellulose, the FTIR spectrums of BC and typing paper (i.e., plant cellulose) were analyzed. Since the BC was difficult to be powdered, the ATR mode with 32 scans per measurement between 400 and $4000 \mathrm{~cm}^{-1}$ was used in this study according to previous studies. ${ }^{34,35}$ As shown in Table 1 and Fig. 6A, the W1-produced BC contained 17 functional groups, most of which were similar to those that in typing paper (Fig. 6B) and the BC produced by other bacteria. ${ }^{36,37}$ For example, the typical wavenumbers of $\mathrm{BC}$ produced by Komagataeibacter sp. W1 (i.e., 1426, 1335, 1314, 1160, 1108, 1054 and $1030 \mathrm{~cm}^{-1}$ ) were also found in A. xylinum ATCC 10245 (i.e., 1426, 1314, 1160 and $1053 \mathrm{~cm}^{-1}$ ), G. xylinus BCRC12335 (i.e., 1335, 1163, 1111, 1060 and $1035 \mathrm{~cm}^{-1}$ ) and $K$. saccharivorans PE5 (i.e., 1425, 1319, 1157 and $1023 \mathrm{~cm}^{-1}$ ). ${ }^{38,39}$

Specifically, the typical absorption at around $3300 \mathrm{~cm}^{-1}$ (peak 1) corresponds to stretching vibration of intra and inter $\mathrm{O}-\mathrm{H}$ in cellulose $\mathrm{I}^{\mathbf{3 4 , 4 0 , 4 1}}$ While the weak peak at around $2900 \mathrm{~cm}^{-1}$ (peaks 2 and 3 ) corresponds to $\mathrm{C}-\mathrm{H}$ stretching of $\mathrm{CH}_{2}$ and $\mathrm{CH}_{3}$ groups or $\mathrm{CH}_{2}$ asymmetric stretching (Fig. 6), the latter is often found in plant cellulose (Fig. $6 \mathrm{~B}){ }^{2,34}$ For both BC and typing paper, a peak at around $1650 \mathrm{~cm}^{-1}$ (peak 4) is observed, corresponding to $\mathrm{H}-\mathrm{O}-\mathrm{H}$ bending of absorbed water. $^{2,40}$ Although it is thought that the peak at around $1430 \mathrm{~cm}^{-1}$ (peak 5) may originate from $\mathrm{CH}_{2}$ scissoring, ${ }^{42}$ most studies attribute it to $\mathrm{CH}_{2}$ symmetric bending or $\mathrm{O}-\mathrm{H}$ in plane bending. ${ }^{2,43}$ The peak at around $1360 \mathrm{~cm}^{-1}$ corresponds to $\mathrm{C}-\mathrm{H}$

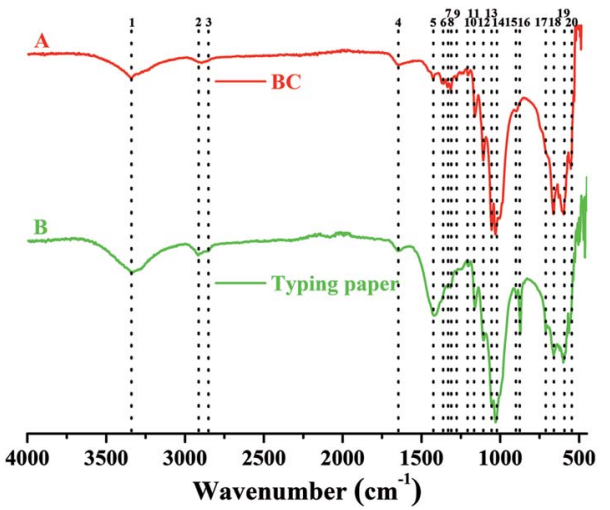

Fig. 6 FTIR analysis of W1-produced bacterial cellulose on a Nicolet iS5 in the ATR mode with 32 scans per measurement between 400 and $4000 \mathrm{~cm}^{-1}$.

bending, which also results in a typical absorption at 1280 and $1204 \mathrm{~cm}^{-1}$ (Fig. 6A). ${ }^{9,29,43}$ While the peak at $1335 \mathrm{~cm}^{-1}$ (peak 7) may correspond to $\mathrm{C}-\mathrm{H}$ deformation or $\mathrm{O}-\mathrm{H}$ in-plane bending, ${ }^{2,9}$ the absorption at around $1315 \mathrm{~cm}^{-1}$ (peak 8) is assigned to out-of-plane wagging of the $\mathrm{CH}_{2}$ groups. ${ }^{42}$ As a typical indicator of the presence of $\mathrm{C}-\mathrm{O}-\mathrm{C}$ antisymmetric bridge stretching of $1,4-\beta$-D-glucoside in $\mathrm{BC}$, the absorption at around $1160 \mathrm{~cm}^{-1}$ is also observed. ${ }^{2,36}$ It has shown that the peaks at $1000-1100 \mathrm{~cm}^{-1}$ can be assigned to $\mathrm{C}-\mathrm{O}$ stretching vibration in primary alcohol and $\mathrm{C}-\mathrm{O}-\mathrm{C}$ skeletal vibration. ${ }^{2,36,43}$ However, these hypotheses are controversial. For example, some studies attribute the absorption at 1030 and $1054 \mathrm{~cm}^{-1}$ to the bending of $\mathrm{C}-\mathrm{O}-\mathrm{H}$ bond of carbohydrates ${ }^{4-46}$ or $\mathrm{C}-\mathrm{O}-\mathrm{C}$

Table 1 Comparative analysis of functional groups on BC and typing paper by FTIR

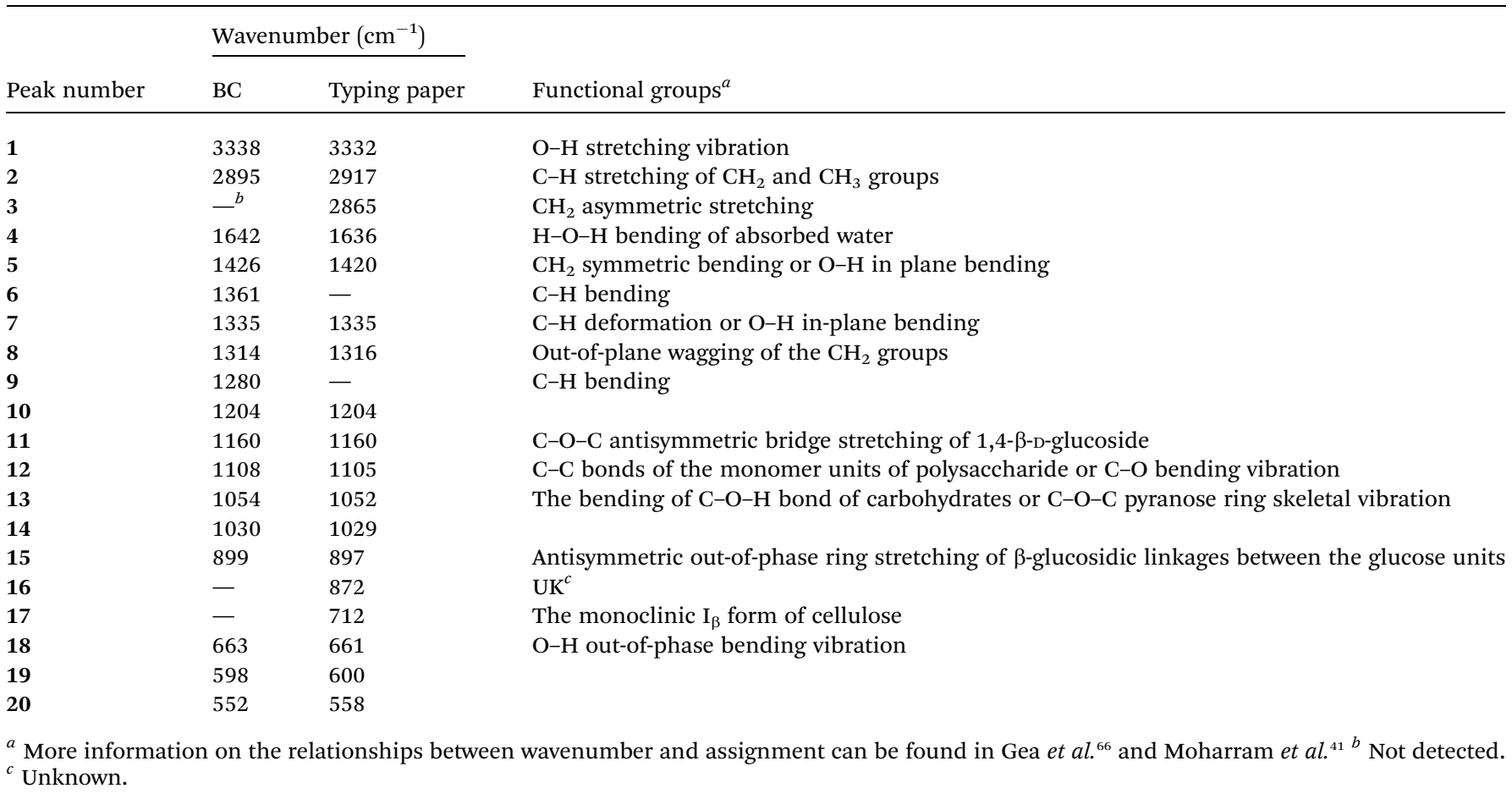


pyranose ring skeletal vibration. ${ }^{\mathbf{1 , 4 0 , 4 6}}$ Moreover, Gao et al. ${ }^{\mathbf{4 7}}$ showed that the peak at $1030 \mathrm{~cm}^{-1}$ might also be associated with the presence of $\mathrm{OCH}_{3}$, while the peak at $1108 \mathrm{~cm}^{-1}$ indicated $\mathrm{C}-\mathrm{C}$ bonds of the monomer units of polysaccharide ${ }^{45}$ or $\mathrm{C}-\mathrm{O}$ bending vibration, ${ }^{9}$ i.e., skeletal vibration. Furthermore, the peaks at 1162, 1107 and $1055 \mathrm{~cm}^{-1}$ can be assigned to $\mathrm{C}-\mathrm{C}$ stretching vibration, skeletal vibration and ring vibration, respectively. ${ }^{48}$ Besides, we also found four peaks at $400-$ $1000 \mathrm{~cm}^{-1}$ (Fig. 6A). Among of which, the peak at around $900 \mathrm{~cm}^{-1}$ corresponds to antisymmetric out-of-phase ring stretching of $\beta$-glucosidic linkages between the glucose units, , $^{2,439}$ which is designated as an "amorphous" absorption band, mainly contributing to the increase of BC intensity. ${ }^{\mathbf{4 5 , 5 0}}$ For the peak at $712 \mathrm{~cm}^{-1}$, it is assigned to the monoclinic $I_{\beta}$ form of plant cellulose, ${ }^{9}$ but which is absent in BC (Fig. 6). Unlike the peak 17, the peaks 18-20 are present in both BC and typing paper, corresponding to $\mathrm{O}-\mathrm{H}$ out-of-phase bending vibration. $^{2}$

Since two weak peaks occur at around 1430 and $900 \mathrm{~cm}^{-1}$ (Fig. 6A), we hypothesized that the BC produced by Komagataeibacter sp. W1 mainly consisted of pure cellulose $\mathrm{I}^{\mathbf{5 0 - 5 2}}$ which was in accordance with XRD data (Fig. 5). Other peaks corresponding to cellulose I are also found in this study, e.g., 3338, 1160 and $899 \mathrm{~cm}^{-1}$, which have been well studied previously. ${ }^{53,54}$ However, another three weak peaks at $\mathbf{1 3 3 5}$, 1314 and $1280 \mathrm{~cm}^{-1}$ and the blue-shift of wavenumber from 1430 to $1426 \mathrm{~cm}^{-1}$ give the evidence of the presence of cellulose II..$^{53,54}$

\subsection{Genome sequence identification in BC-producing} bacterial isolate for cellulose synthesis and regulation

It is well-known that the $\mathrm{BC}$ production in bacteria is associated with several enzymes, including glucokinase (EC 2.7.1.2), phosphoglucomutase (EC 5.4.2.2), UTP-glucose-1-phosphate uridylyltransferase (EC 2.7.7.9) and cellulose synthases (EC 2.4.1.12). ${ }^{23}$ However, these enzymes vary in different genera of BC-producing bacteria. ${ }^{55}$ Moreover, the complete genomes of Komagataeibacter have only been sequenced in four species such as $K$. nataicola RZS01, K. xylinus E25, K. hansenii ATCC 23769 and $K$. medellinensis NBRC3288, while draft genomes are available for $K$. europaeus LMG18494, $K$. intermedius AF2, $K$. rhaeticus AF1, K. kakiaceti JCM 25156 and $K$. oboediens 174Bp2.6,16,56,57 To have an overview of the relevant enzymes in Komagataeibacter sp. W1, we also sequenced the draft genome and analyzed the functional genes and signaling pathways associated with BC synthesis.

After removing the low-quality sequences, the total bases of clean data were about $1.32 \mathrm{~Gb}$ (Table $\mathrm{S} 1 \dagger$ ). The genome assembly showed that there were 168 scaffolds and 285 contigs in the draft genome (Table $\mathrm{S} 2 \dagger$ ). Moreover, a total of 3705 genes (i.e., open reading frames, orfs) were found based on sequence alignment with the known information in the KEEG database (Tables S3 and S4†). However, only 1783 and 1732 out of 3705 genes were annotated to COG functional categories and certain KEEG pathways, respectively (Fig. 7 and Table S5†). As shown in Fig. 7, there were 113 genes that corresponded to carbohydrate transport and metabolisms, of which 3 orfs were responsible for encoding glucokinase, phosphoglucomutase and UTP-glucose-

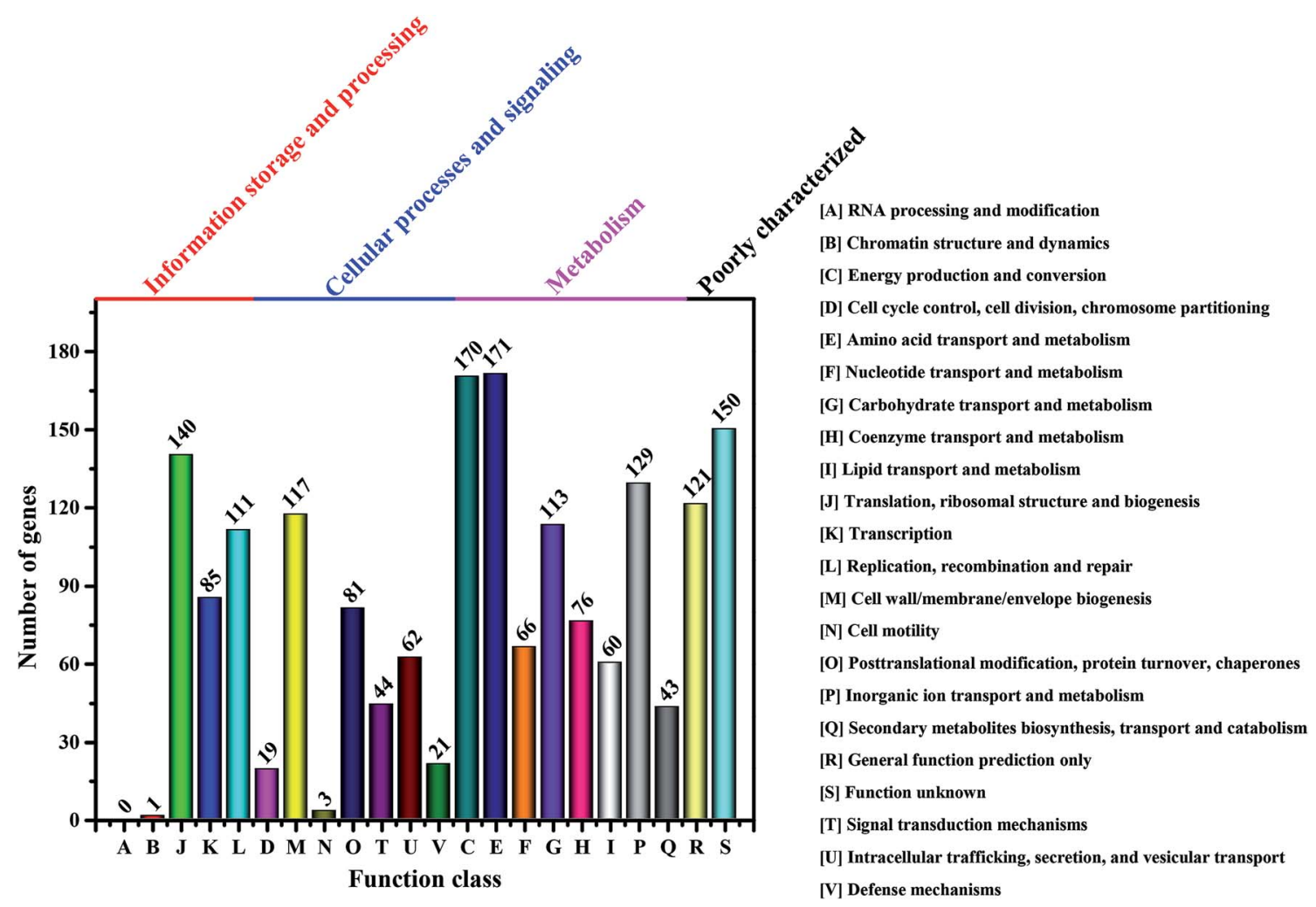

Fig. 7 Number of genes associated with general COG functional categories based on the total number of protein coding genes in the genome. 


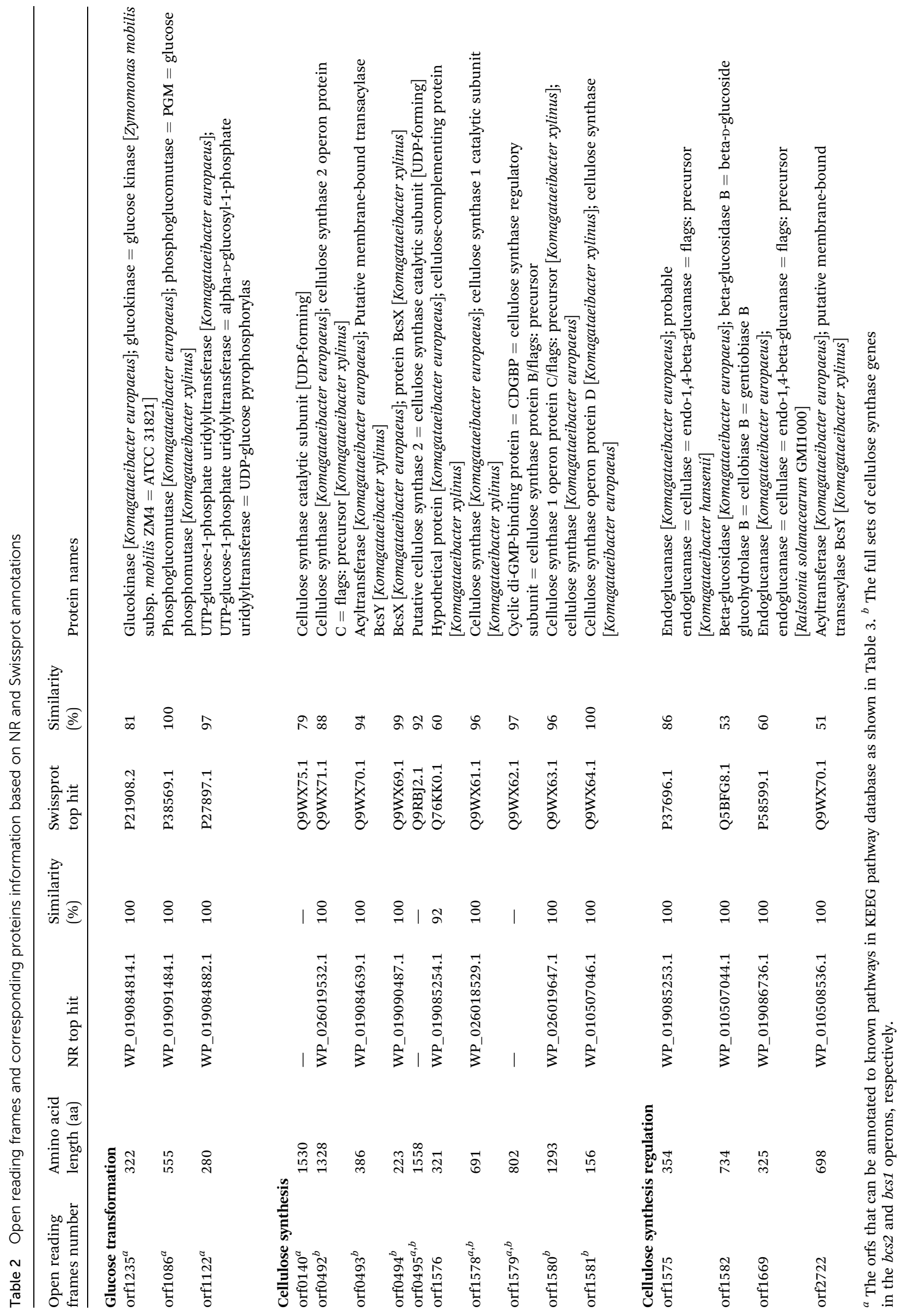


1-phosphate uridylyltransferase, respectively, and 14 orfs were responsible for encoding cellulose synthases and associated coenzymes (Table 2).

By KEEG analysis, the enzymes encoded by orfs 1235, 1086 and 1122 were involved in glucose transformation in starch and sucrose metabolism pathway (Table 3 and Fig. S1†). All these enzymes co-produced the precursor (i.e., UDP-glucose) of $\mathrm{BC}$ (Fig. 8A), which was in accordance with the documented studies. ${ }^{23,27}$ Once UDP-glucose produced, the domain A encoded by $b c s A$ and attached inner cell membrane is thought to catalyze the UDP-glucose to cellulose. ${ }^{27}$ This process also needs another core enzyme, i.e., BcsB, to accelerate the $\mathrm{BC}$ synthesis by combining to c-di-GMP. ${ }^{27,58,59}$ After that, the newly synthesized $\mathrm{BC}$ is crystallized with the aid of BcsD and then extruded by
BcsC (Fig. 8B and C). ${ }^{60}$ As expected, all the genes associated with above proteins encoding were detected in the strain $\mathrm{W} 1$ and the corresponding amino acid sequences shared high similarity with $K$. europaeus and $K$. xylinus (Table 2). However, both BcsC and BcsD could not be annotated to certain KEEG pathways (Table 3). As stated by previous studies, these genes are located in bcs operon1 (bcs1). ${ }^{6,27}$ Similar to Zhang et al. ${ }^{6}$ we also found two upstream genes cmcax (orf1575) and ccpAx (orf1576), and one downstream gene bglxA (orf1582) in bcs1 (Table 2 and Fig. 8A). While ccpAx encodes a cellulose-complementing protein, ${ }^{61,62}$ cmcax and $b g l x A$ encode endo- $\beta-1,4$-glucanase (EC 3.2.1.4) and $\beta$-glucosidase (EC 3.2.1.21), respectively, both of which assist cellulose biosynthesis by hydrolysing tangled glucan chains when a failure in chain arrangement occurs

Table 3 Open reading frames and corresponding proteins information based on KEEG pathway annotation

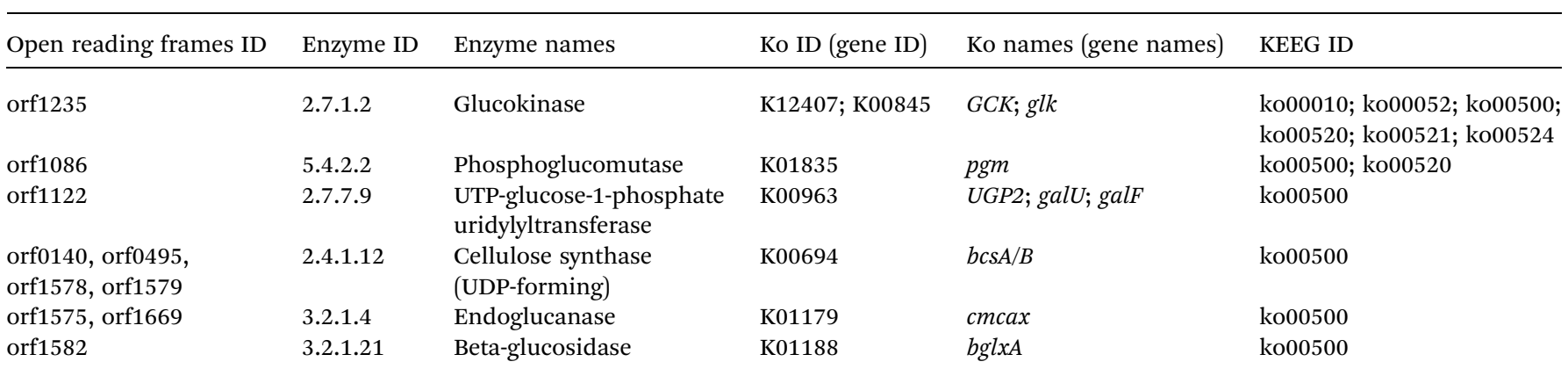
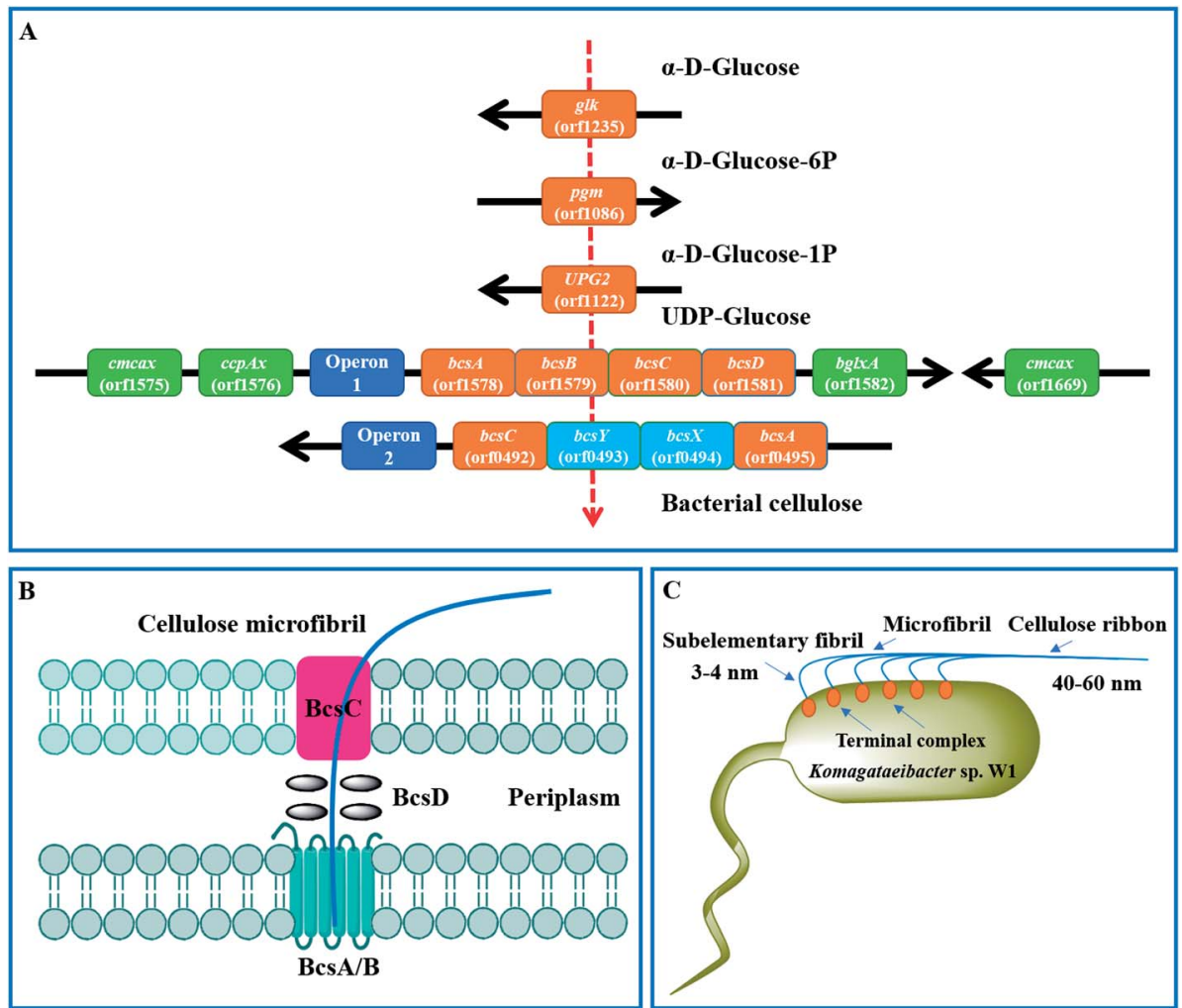

Fig. 8 Mechanisms of BC synthesis in Komagataeibacter sp. W1. (A) genes associated with glucose transformation and BC synthesis; (B) proposed $\mathrm{BC}$ synthesis and excretion pathways by the regulation of bcs operon1; ${ }^{60}$ (C) BC assembly programs. ${ }^{27}$ 
(Fig. S2 $\dagger$ ). ${ }^{6,63}$ It was interesting to note that another cmcax gene (orf 1669) was also found in Komagataeibacter sp. W1 (Table 2), which was different from $K$. nataicola ${ }^{6}$ but similar to $K$. europaeus (Table 2) and its functions in $\mathrm{BC}$ synthesis regulation warranted further studies. As shown in Table 2 and Fig. 8A, another $b c s$ operon ( $b c s 2$ ) for BC synthesis was also found in this study. The bcs 2 was composed of $b c s A$ (orf0495), $b c s X$ (orf0494), $b c s Y$ (orf0493) and $b c s C$ (orf0492), of which $b c s X$ and $b c s Y$ were located in the middle of $b c s A$ and $b c s C$ as previous report, ${ }^{6}$ but their potential roles in $\mathrm{BC}$ synthesis remained unknown. Morgan et $a l .{ }^{64}$ showed that the protein encoded by bcsA contained the catalytically active subunit with a Pilz domain, which was responsive to c-di-GMP. Similar to Zhang et al., ${ }^{6}$ we also found another bcsA (orf0140), which might be located on the reverse strand and its encoded protein did not contain a PilZ domain. Except for above genes, another $b c s Y$ (orf2722) was also located in the genome of Komagataeibacter sp. W1, which might involve in the expression of acyltransferase (Table 2).

As described, the Pilz domain is in response to the second messenger c-di-GMP. c-di-GMP is produced from 2 molecules of GTP by diguanylate cyclases (DGCs) and is broken down into $5^{\prime}$ phosphoguanylyl-( $\left.3^{\prime}-5^{\prime}\right)$-guanosine by specific phosphodiesterases (PDEs). ${ }^{6}$ The c-di-GMP level can stimulate BC synthesis, leading to a 100 -fold of BC increase.${ }^{65}$ Similar to Zhang et al. ${ }^{6}$ we found three $c d g$ operons containing a c-di-GMP PDE gene (orf2263, orf2943 and orf3022) followed by a DGC gene (orf2264, orf2944 and orf3023). Besides, four standalone c-di-GMP PDE genes (orf1651, orf1917 and orf1918), one standalone cAMP DPE gene (orf2811) and three standalone DGC genes (orf0413, orf1556 and orf 2256) were also located in the genome of Komagataeibacter sp. W1 (Table S4 $\dagger$ ). Given the presence of two bcs operons in BC synthesis and three $c d g$ operons in c-di-GMP regulation, it was possible that all these operons were attributed to the great ability of BC biosynthesis in Komagataeibacter sp. W1.

\section{Conclusions}

In this study, a BC-producing bacterium namely Komagataeibacter sp. W1 was isolated from a fermented vinegar. The SEM, XRD and FTIR analyses revealed that the pure BC produced by W1 was mostly cellulose I and composed of nanofibrils. By a draft genome sequencing, all typical genes associated with $\mathrm{BC}$ synthesis and regulation were found. However, the functional verification of some genes that might involve in BC synthesis coregulation warranted future research.

\section{Conflicts of interest}

There are no conflicts to declare.

\section{Acknowledgements}

This work was supported in party by the Science and Technology Program of Fujian Province (2017Y0027), the Education Department Fund of Fujian Province (JAT170144), the Research Project of Fujian Normal University (No. 5c1005), the Special Fund of Quangang Petrochemical Research Institute of Fujian
Normal University (2016YJY01) and the Hong-Wu Weng Original Fund of Peking University.

\section{References}

1 C. Castro, A. Vesterinen, R. Zuluaga, G. Caro, I. Filpponen, O. J. Rojas, G. Kortaberria and P. Gañán, Cellulose, 2014, 21, 1745-1756.

2 A. Ashori, S. Sheykhnazari, T. Tabarsa, A. Shakeri and M. Golalipour, Carbohydr. Polym., 2012, 90, 413-418.

3 H. P. S. A. Khalil, A. H. Bhat and A. F. I. Yusra, Carbohydr. Polym., 2012, 87, 963-979.

4 Y. Nishiyama, J. Wood Sci., 2009, 55, 241-249.

5 S. M. A. S. Keshk and A. F. El-Kott, in Science and Principles of Biodegradable and Bioresorbable Medical Polymers, ed. X. C. Zhang, Woodhead Publishing is an imprint of Elsevier, United Kingdom, 2017, pp. 295-319.

6 H. Zhang, X. Xu, X. Chen, F. Yuan, B. Sun, Y. Xu, J. Yang and D. Sun, Sci. Rep., 2017, 7, 4431.

7 I. Reiniati, A. N. Hrymak and A. Margaritis, Crit. Rev. Biotechnol., 2017, 37, 510-524.

8 H. K. Uzyol and M. T. Saçan, Environ. Sci. Pollut. Res., 2017, 24, 11154-11162.

9 C. Castro, R. Zuluaga, J.-L. Putaux, G. Caro, I. Mondragon and P. Gañán, Carbohydr. Polym., 2011, 84, 96-102.

10 R. V. Augimeri, A. J. Varley and J. L. Strap, Front. Microbiol., 2015, 6, 1282.

11 K. Komagata, T. Iino and Y. Yamada, in The Prokaryotes, ed. E. Rosenberg, E. F. DeLong, S. Lory, E. Stackebrandt and F. Thompson, Springer Berlin Heidelberg, Germany, Berlin, 2014, pp. 3-78.

12 F. Barja, C. Andrés-Barrao, R. O. Pérez, E. M. Cabello and M.-L. Chappuis, in Acetic Acid Bacteria, ed. K. Matsushita, H. Toyama, N. Tonouchi and A. Okamoto-Kainuma, Springer, Japan, 2016, pp. 201-221.

13 M. J. Valera, M. J. Torija, A. Mas and E. Mateo, Appl. Microbiol. Biotechnol., 2015, 99, 1349-1361.

14 Y. Yamada, Int. J. Syst. Evol. Microbiol., 2014, 64, 1670-1672. 15 Y. Yamada, P. Yukphan, H. T. L. Vu, Y. Muramatsu, D. Ochaikul, S. Tanasupawat and Y. Nakagawa, J. Gen. Appl. Microbiol., 2012, 58, 397-404.

16 M. J. Valera, A. Poehlein, M. J. Torija, F. S. Haack, R. Daniel, W. R. Streit, E. Mateo and A. Mas, Genome Announc., 2015, 3, e01231-15.

17 M. J. Valera, A. Mas, W. R. Streit and E. Mateo, Microb. Cell Fact., 2016, 15, 88.

18 R. Jayabalan, R. V. Malbaša, E. S. Lončar, J. S. Vitas and M. Sathishkumar, Compr. Rev. Food Sci. Food Saf., 2014, 13, 538-550.

19 J. K. Park, Y. H. Park and J. Y. Jung, Biotechnol. Bioprocess Eng., 2003, 8, 83-88.

20 S.-S. Wang, S.-L. Ye, Y.-H. Han, X.-X. Shi, D.-L. Chen and M. Li, RSC Adv., 2016, 6, 101153-101161.

21 S. Hestrin and M. Schramm, Biochem. J., 1954, 58, 345-352. 22 R. Li, H. Zhu, J. Ruan, W. Qian, X. Fang, Z. Shi, Y. Li, S. Li, G. Shan, K. Kristiansen, S. Li, H. Yang, J. Wang and J. Wang, Genome Res., 2010, 20, 265-272. 
23 I. M. Saxena and J. R. Malcolm Brown, in Bacterial NanoCellulose-A Sophisticated Multifunctional Material, ed. M. Gama, P. Gatenholm and D. Klemm, CRC Press, USA, Boca Raton, Florida, 2013, pp. 1-18.

24 Y. Nishi, M. Uryu, S. Yamanaka, K. Watanabe, N. Kitamura, M. Iguchi and S. Mitsuhashi, J. Mater. Sci., 1990, 25, 29973001.

25 S. K. Cousins and R. M. Brown Jr, Polymer, 1995, 36, 38853888.

26 S. K. Cousins and R. M. Brown Jr, Polymer, 1997, 38, 903-912.

27 Y. Amano, F. Ito and T. Kanda, J. Biol. Macromol., 2005, 5, 310.

28 S. Yamanaka, K. Watanabe, N. Kitamura, M. Iguchi, S. Mitsuhashi, Y. Nishi and M. Uryu, J. Mater. Sci., 1989, 24, 3141-3145.

29 M. Ul-Islam, J. H. Ha, T. Khan and J. K. Park, Carbohydr. Polym., 2013, 92, 360-366.

30 H. Luo, G. Xiong, Z. Yang, S. R. Raman, H. Si and Y. Wan, RSC Adv., 2014, 4, 14369-14372.

31 M. Wada and T. Okano, Cellulose, 2001, 8, 183-188.

32 A. C. O'Sullivan, Cellulose, 1997, 4, 173-207.

33 K. Watanabe, M. Tabuchi, Y. Morinaga and F. Yoshinaga, Cellulose, 1998, 5, 187-200.

34 A. Tercjak, J. Gutierrez, H. d. S. Barud, R. R. Domeneguetti and S. J. L. Ribeiro, ACS Appl. Mater. Interfaces, 2015, 7, 4142-4150.

35 H. Sai, R. Fu, L. Xing, J. Xiang, Z. Li, F. Li and T. Zhang, ACS Appl. Mater. Interfaces, 2015, 7, 7373-7381.

36 M. C. I. M. Amin, N. Ahmad, N. Halib and I. Ahmad, Carbohydr. Polym., 2012, 88, 465-473.

37 L. Hong, Y. L. Wang, S. R. Jia, Y. Huang, C. Gao and Y. Z. Wan, Mater. Lett., 2006, 60, 1710-1713.

38 E. A. Hassan, H. M. Abdelhady, S. S. A. El-Salam and S. M. Abdullah, Br. Microbiol. Res. J., 2015, 9, 1-13.

39 H.-H. Chen, L.-C. Chen, H.-C. Huang and S.-B. Lin, Cellulose, 2011, 18, 1573-1583.

40 F. Yassine, N. Bassil, A. Chokr, A. E. Samrani, A. Serghei, G. Boiteux and M. E. Tahchi, Cellulose, 2016, 23, 1087-1100.

41 M. A. Moharram and O. M. Mahmoud, J. Appl. Polym. Sci., 2008, 107, 30-36.

42 S. Dammström, L. Salmén and P. Gatenholm, Polymer, 2005, 46, 10364-10371.

43 H. S. Barud, J. L. Souza, D. B. Santos, M. S. Crespi, C. A. Ribeiro, Y. Messaddeq and S. J. L. Ribeiro, Carbohydr. Polym., 2011, 83, 1279-1284.

44 D. Sun, J. Yang and X. Wang, Nanoscale, 2010, 2, 287-292.

45 M. S. Dayal, N. Goswami, A. Sahai, V. Jain, G. Mathur and A. Mathur, Carbohydr. Polym., 2013, 94, 12-16.
46 C. Huang, X.-Y. Yang, L. Xiong, H.-J. Guo, J. Luo, B. Wang, H.-R. Zhang, X.-Q. Lin and X.-D. Chen, Lett. Appl. Microbiol., 2015, 60, 491-496.

47 W.-H. Gao, K.-F. Chen, R.-D. Yang, F. Yang and W.-J. Han, BioResources, 2011, 6, 144-153.

48 D. Li, K. Ao, Q. Wang, P. Lv and Q. Wei, Molecules, 2016, 21, 618.

49 T. Kondo and C. Sawatari, Polymer, 1996, 37, 393-399.

50 W. N. Goh, A. Rosma, B. Kaur, A. Fazilah, A. A. Karim and R. Bhat, Int. Food Res. J., 2012, 19, 153-158.

51 X. Feng, N. Ullah, X. Wang, X. Sun, C. Li, Y. Bai, L. Chen and Z. Li, J. Food Sci., 2015, 80, E2217-E2227.

52 M. L. Nelson and R. T. O'Connor, J. Appl. Polym. Sci., 1964, 8, 1311-1324.

53 M. U. Rani, N. K. Rastogi and K. A. A. Appaiah, J. Microbiol. Biotechnol., 2011, 21, 739-745.

54 M. U. Rani, K. Udayasankar and K. A. A. Appaiah, J. Appl. Polym. Sci., 2011, 120, 2835-2841.

55 B. L. Quéré and J.-M. Ghigo, Mol. Microbiol., 2009, 72, 724740.

56 R. A. C. d. Santos, A. A. Berretta, H. d. S. Barud, S. J. L. Ribeiro, L. N. González-García, T. D. Zucchi, G. H. Goldman and D. M. Riaño-Pachón, Genome Announc., 2015, 3, e01404-15.

57 R. A. C. d. Santos, A. A. Berretta, H. d. S. Barud, S. J. L. Ribeiro, L. N. González-García, T. D. Zucchi, G. H. Goldman and D. M. Riaño-Pachón, Genome Announc., 2014, 2, e00731-14.

58 O. Omadjela, A. Narahari, J. Strumillo, H. Mélida, O. Mazur, V. Bulone and J. Zimmer, Proc. Natl. Acad. Sci. U. S. A., 2013, 110, 17856-17861.

59 J. B. McManus, Y. Deng, N. Nagachar, T.-h. Kao and M. Tien, Enzyme Microb. Technol., 2016, 82, 58-65.

60 P. R. Iyer, J. Catchmark, N. R. Brown and M. Tien, Cellulose, 2011, 18, 739-747.

61 S. Kawano, K. Tajima, Y. Uemori, H. Yamashita, T. Erata, M. Munekata and M. Takai, DNA Res., 2002, 9, 149-156.

62 N. Sunagawa, T. Fujiwara, T. Yoda, S. Kawano, Y. Satoh, M. Yao, K. Tajima and T. Dairi, J. Biosci. Bioeng., 2013, 115, 607-612.

63 T. Nakai, Y. Sugano, M. Shoda, H. Sakakibara, K. Oiwa, S. Tuzi, T. Imai, J. Sugiyama, M. Takeuchi, D. Yamauchi and Y. Mineyuki, J. Bacteriol., 2013, 195, 958-964.

64 J. L. W. Morgan, J. Strumillo and J. Zimmer, Nature, 2013, 493, 181-186.

65 P. Ross, R. Mayer and M. Benziman, Microbiol. Mol. Biol. Rev., 1991, 55, 35-58.

66 S. Gea, C. T. Reynolds, N. Roohpour, B. Wirjosentono, N. Soykeabkaew, E. Bilotti and T. Peijs, Bioresour. Technol., 2011, 102, 9105-9110. 